\title{
An automatic crude registration of two partially overlapping range images
}

\author{
Mercedes R. Gonzales Márquez \\ Computer Science Course \\ State University of Mato Grosso do Sul \\ 79804-970 - Dourados, MS, Brazil \\ mercedes@uems.br
}

\author{
Wu, Shin - Ting \\ School of Electrical and Computer Engineering - DCA \\ University of Campinas - UNICAMP \\ 13083-970, Campinas, SP, Brazil \\ ting@dca.fee.unicamp.br
}

\begin{abstract}
We present a crude registration algorithm for two partially overlapping range images acquired from a rigid object. Our proposal is characterized by two novel features: drastic data reduction and expressive shape descriptor. The data reduction is achieved on the basis of the observation that for crude alignments only the overall shape of image is of interest and the enhancement of the expression power of the shape descriptor is accomplished by integrating in it both local and global shape information. Experimental results show that our algorithm delivers good initial estimate to a fine registration program.
\end{abstract}

\section{Introduction}

With the advent of inexpensive 3D model acquisition systems, it is increased the demand for a robust way to assemble range images captured from multiple perspectives into one coordinate system in order to build a complete 3D model. Figure 1 illustrates the registration of two range images, the target image $S$ and the reference image $D$, acquired from two different points-of-view defined in proper coordinate references.

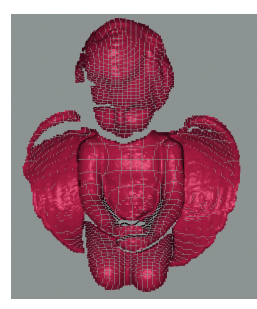

(a) angel $_{1}$

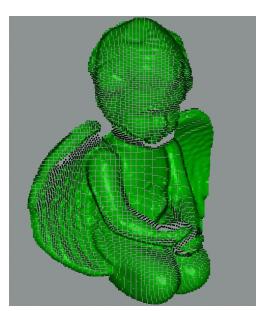

(b) angel 2

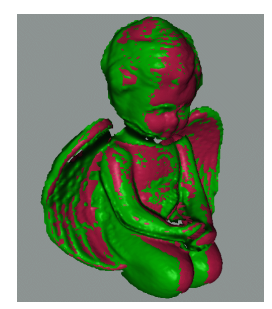

(c) 3D model
Figure 1. Range images of an angel: (a) $S$ image; (b) $D$-image; (c) integrated model.
Without a priori knowledge of the relative displacements of the acquisition device, the crude registration is essential for a registration of partially overlapped images. Assuming that the poses only differ in a rigid transformation $\mathcal{T}$, a crude registration consists in providing an initial guess $\mathcal{T}_{0}$ which should be reasonably close to $\mathcal{T}$. Then, in a fine registration step $\mathcal{T}_{0}$ is tuned to $\mathcal{T}$ by minimizing the pointto-point distances or the extrinsic errors.

In the last decade, some matching algorithms on the basis of presumed pairs of correspondences have been proposed for replacing tedious manual crude alignments, in which users directly choose pairs of correspondences necessary for defining an appropriate $\mathcal{T}$. Most of proposed crude registration methods search for the best solution among the pairs of correspondences that fulfil some matching criteria. They differ basically in the way that they search for a solution and the matching criteria that they employ. The matching criteria may be invariant geometric features, structures, and/or textures.

In this paper we present a novel way to efficiently select all pairs of elements in the reference and the target images that fulfil given matching criteria. Instead of a dense data set, we propose to use a sparse data set, whose shape resembles the original one. This is because that an object may be modeled as a (possibly infinite) point set, but its shape is essentially captured by a mesh of a finite subset [3]. Figure 2 illustrates four meshes with distinct decimation levels (or distinct approximation error $\epsilon$ given in terms of distance $s$ explained in Section 3), but suggesting the same physical object. To validate our proposal, we need a decimation algorithm that preserves the overall shape. We successfully apply the QSLIM simplification algorithm [9]. It clusters the samples according to a planarity condition, as detailed in Section 3.

On the basis of the fact that an affinity in $\Re^{3}$ is uniquely determined by its effect on any one tetrahedron, we also propose an original shape descriptor that geometrically synthesizes our matching criteria: a tetrahedron. Since the four vertices of the tetrahedron correspond to four non-coplanar 


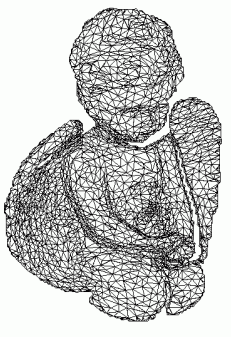

(a) $\epsilon=0.08 \mathrm{~s}$

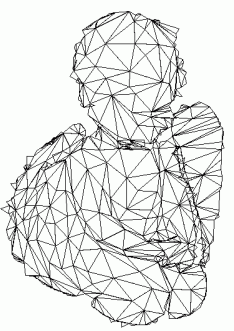

(c) $\epsilon=0.75 \mathrm{~s}$

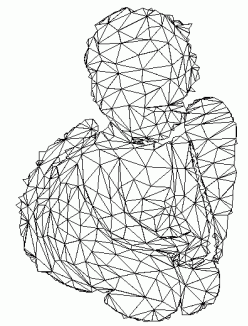

(b) $\epsilon=0.5 \mathrm{~s}$

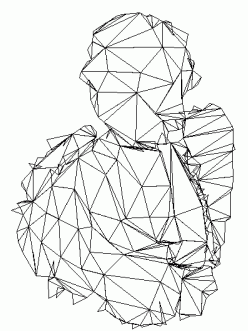

(d) $\epsilon=s$
Figure 2. Several decimation levels

spatially dispersed samples in the decimated mesh, it conveys the local features (of the four samples) and the overall structure of the decimated mesh (the relative position of the samples). In Section 4 we present a procedure to construct our proposed shape descriptor. Representing the matching criteria as a geometric entity, we may apply the conjunctive searching strategy proposed by Chen et al. [7] to find all matchable tetrahedrons in the reference image. In fact, we extended their algorithm from $2 \mathrm{D}$ to $3 \mathrm{D}$, as explained in Section 5.

In Section 6 the results that validate our proposal are presented. We performed the analysis of our proposed methods with respect to its robustness to high decimation level and its robustness to low overlapping region. Finally, in section 7 some concluding remarks are given.

\section{Related Works}

The algorithms for crude registration of rigid models work either on the basis of the geometric measurements of the whole sample points with regard to a small point set, or on the basis of the geometrical characteristics of the vicinity of this small point set. As far as we know, they work on the dense original range data.

In Spin-image matching (SIM) procedure, a small number of oriented points is randomly chosen from $S$-image [1]. From each of those points, a spin-image is generated, consisting of a 2D-histogram constructed by the radial distance (to the surface normal at the oriented point) and the ax- ial distance (to the tangent plane at the point) of all other points. Then, spin-images associated to distinct points of the $D$-image are compared with a few sets of the previously built spin-images for constructing matched pairs and estimating transformation candidates. False transformations are pruned by the ICP algorithm. According to the careful comparisons carried out by Planitz et al., this technique may take a long time and require excessive storage for high resolution surfaces [5]. Moreover, it may fail when the models are axis-symmetric [6]. Our proposed method not only has less storage complexity but is suitable for axis-symmetric models, as well.

Instead of histograms constructed by the radial and the axial distances, Geometric histogram matching (GHM) method is based on a few sets of histograms from the triangular mesh of the $S$-image, each of which represents the relative angle between the normal of a given facet $f_{i}$ and all other surrounding facets, within a predefined distance from the plane in which $f_{i}$ lies to all points of the surrounding facets [2]. A match for facet $f_{i}$ is determined by finding the best match between its histograms and the histograms representing the facets of the triangular mesh of the $D$-image. The Random Sample Consensus (RANSAC) algorithm is used to compute the best orientation and the best translation for a plausibly correct final alignment [11]. It was verified that GHM is unsuitable for surfaces with few distinct surface curvature variations [5]. In this work, we show that our proposed method also works well on models with smooth curvature variations, since the mesh is appropriately decimated.

RANSAC-based DARCES (RBD) is an acronym for Random Sample Consensus based Data Aligned Rigidity Constrained Exhaustive Search [7]. Differently from the SIM and the GHM techniques, it does not require the computation of the geometrical characteristics of the whole sample points. In each iteration of the RBD algorithm, a sample point $s_{1}$ is randomly chosen from $S$-image, which together with two nearby non-collinear sample points $s_{2}$ and $s_{3}$ builds a triangular structure $L$. Each sample in $D$-image is then exhaustively tested aiming to find a triangle that is matchable to $L$. For the first point $s_{1}$, any point of $D$ image is considered as a potentially matchable point $m_{1}$. The search region for a corresponding point $m_{2}$ to $s_{2}$ can be limited to the sphere of radius $d_{12}$ centered at $m_{1}$ where $d_{12}$ is the distance between $s_{1}$ and $s_{2}$. And the search region for a corresponding point $m_{3}$ to $s_{3}$ can be further limited to the intersection of two spheres, one centered at $m_{1}$ with radius $d_{13}$ and the other centered at $m_{2}$ with radius $d_{23}$. For evaluating the quality of alignment transformation, the authors use a subsample of the original data. Although it works for a wide variety of surfaces, it may take a long time for finding a good initial estimate that converges to a correct alignment. Instead of a planar structure, we pro- 
pose to use a spatial structure. Because a spatial structure is more distinguishable, fewer matchable structures are selected, which makes feasible to test all of them for getting the best one. Consequently, the outcome of our proposed algorithm is quantitatively and qualitatively better, as shown in Section 6.

In the Intrinsic curve matching (ICM) procedure, curves with zero mean curvature are extracted from range images [10]. For each individual range image, all possible pairings between curves are established. In each pairing, the shortest line segment (connector) is determined in $S$-image and its length is used for finding the best correspondence in $D$-image. The quality of the transformation is evaluated by the number of feature alignments. According to Planitz et al., it works better on surfaces with smooth curvature variations than those with sharp edges [5]. Thus, it fails to perform a good alignment for the pair of images of an object with hard edges. Contrary to the ICM algorithm, our proposed model does not suffer from this restriction because the hard edges are discernible features and they will appear in the decimated mesh.

In the Bitangent curve matching ( $B C M$ ) technique, pairs of bitangent curves, that is the curves that share the same tangent plane, are chosen as an invariant feature [18]. The distance function of the bitangent point pairs in terms of the bitangent curve arclength is used for matching. After a good estimate is found, it should be checked whether left-right symmetric pairs have been correctly matched. Moreover, bitangent curves are very special features and it is not ensured that they are present in every image. As our matching structure is built from four non-coplanar sample points, our procedure is much more generic.

\section{Search Space Reduction}

Let $\mathcal{N}_{S}$ and $\mathcal{N}_{D}$ be the number of samples in the target $(S)$ and the reference $(D)$ images, respectively. Theoretically, for finding the best correspondence, we should evaluate $\mathcal{N}_{S} \times \mathcal{N}_{D}$ possible combinations. Nonetheless, for crude alignments only the overall shape of image is of relevance, excessively detailed models do not necessarily deliver more accurate result. This is because that the objective of a crude registration is to approximately align two or more images on the basis of the overlapped region. We only need some distinguishing features of the images. Following this reasoning, we come to the idea to use the samples of the decimated meshes to find the rigid transformation $\mathcal{T}$ that overlays the $S$-image over the $D$-image. Instead of $\mathcal{N}_{S} \times \mathcal{N}_{D}$, we will work with $N_{S} \times N_{D}$ possibilities, with $N_{S}<<\mathcal{N}_{S}$ and $N_{D}<<\mathcal{N}_{D}$.

Quantitatively, we consider that two decimated meshes preserve the overall shape of the corresponding partially overlapping images, if
- the sample density (number of samples per unit area) in the decimated mesh satisfies a specified tolerance $\tau$ for guaranteeing the existence of correspondences in overlapping areas; and

- the samples in the overlapping region are spatially as close as possible.

There are many effective decimation algorithms, but none of them is designed for crude registration. Because of availability and simplification quality, we adapted the simplification algorithm implemented by Garland [9] for evaluating our proposal in practice. Before presenting the adaptations we made, a brief description of the this algorithm is described.

Essentially, the QSLIM simplification algorithm uses iterative edge contractions on the basis of quadric errors associated to each vertex $v$. The squared distance of $v$ to an adjacent face $k$, represented by $n_{k} \cdot v=d$, may be expressed as

$$
\begin{aligned}
D^{2}(v) & =\left(n_{k}^{t} v+d\right)^{2}=\left(v^{t}+d\right)\left(n_{k}^{t} v+d\right) \\
& =v^{t}\left(n_{k} n_{k}^{t}\right) v+2 d n_{k}^{t} v+d^{2}
\end{aligned}
$$

which may be written in a quadratic form

$$
Q(v)=v^{t} A v+2 b^{t} v+c=D^{2}(v)
$$

by making $A=n_{k} n_{k}^{t}, b=d n_{k}, c=d^{2}$. $Q$ can be defined as a triple $Q=(A, b, c)$ where $A$ is a $3 \times 3$ matrix, $b$ is a 3 -vector, and $c$ is a scalar. The addition of quadrics is performed componentwise: $Q_{i}(v)+Q_{j}(v)=\left(Q_{i}+Q_{j}\right)(v)$ where $\left(Q_{i}+Q_{j}\right)=\left(A_{i}+A_{j}, b_{i}+b_{j}, c_{i}+c_{j}\right)$. The quadric error $E$ associated to $v$ is given in terms of the sum of squared distance to all its adjacent faces

$$
E(v)=\sum_{k} D_{k}^{2}(v)=\sum_{k} Q_{k}(v)=Q(v) .
$$

Observe that the initial quadric error estimate for each vertex is 0 , since it lies in the planes of all its adjacent faces.

Let $e_{i j}$ be edges of a triangular mesh whose extreme vertices are $v_{i}$ and $v_{j}$ and $Q_{i}$ and $Q_{j}$ be their associated quadrics, respectively. In each iteration of the simplification algorithm, the optimal contraction $\bar{v}$ for each edge is computed (Figure 3), such that the quadric error of its substitute $\bar{v}, Q(\bar{v})=Q_{i}(\bar{v})+Q_{j}(\bar{v})$ has the minimal quadric error. Since $Q(\bar{v})$ is quadratic, finding its minimum is a linear problem. The minimum occurs where $\partial Q / \partial x=\partial Q / \partial y=$ $\partial Q / \partial z=0$

$$
\Delta Q(v)=2 A v+2 b=0
$$

Assuming that $A$ is invertible, one gets $\bar{v}=-A^{-1} b$. Among all the computed quadric errors, the edge that presents the lowest quadric error is selected to be contracted and the quadric errors of the adjacent vertices are updated. 

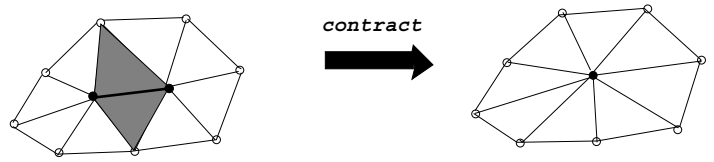

Figure 3. Edge contraction.

The process is repeated, until the desired level of simplification is reached. This simplification process satisfies our filtering requirement, which is to "blur" the geometric properties, or to cluster the samples into a vertex, such that a quadric error of the substitute is less than the specified tolerance $\epsilon$, as illustrates Figure 2.

Nevertheless, if the model is simplified to a very low level of detail, we may miss the minimal number of samples necessary for crude registration, as shown in Figure 4. We should, therefore, establish a minimal level of detail that guarantees acceptable sample density. From our exhaustive tests with all images available in the repositories $[12,16,15]$, we reach a value to the approximation error $\epsilon$ in function of the minimal distance $s$ between two adjacent samples in the original flat range image. It is $\epsilon=s$.

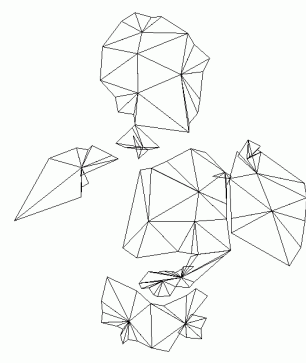

(a) View angle: $0^{\circ}$

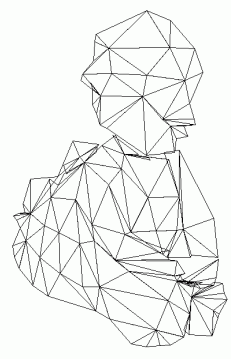

(b) View angle: $40^{\circ}$

\section{Figure 4. Poor approximation $(\epsilon=2)$ leads to loss of correspondences.}

With the approximation error $\epsilon=s$, we observed that for images of smooth shapes the procedure may fail. The problem comes from the fact that the decimation procedure is applied independently in the $S$-mesh and the $D$-mesh. Hence, the contraction vertices in the overlapping region may not overlap, as highlighted in the inset of Figure 5. From the inset we may observe that the remaining vertices of the decimated mesh drawn in thinner lines (Figure 5.a) do not overlap the vertices of the mesh drawn in thicker lines (Figure 5.b). If we increase the sample density, the probability of samples being almost overlapped also increase. Further specific tests with this class of images lead us to another relation: $\epsilon=0.15 \mathrm{~s}$. The error approximation should, therefore, be dependent on the image geometry. It should ensure not only the minimal number of samples, but also that the samples in overlapping regions are always matchable as well. Our proposed rule of thumb for calibrating the approximation error with respect to $s$ is: $0.15 s$ for images with almost planar features; $s$ for images with several prominent features; and $0.5 \mathrm{~s}$ for the in-between images. Adopting this rule we have achieved, in average, a reduction factor of $95 \%$ with respect to the original data.

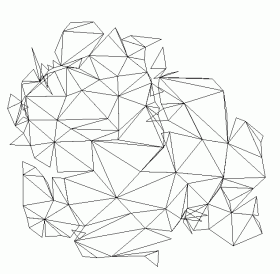

(a) Angle: $0^{\circ}$

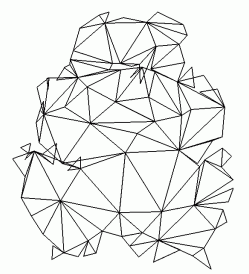

(b) Angle: $40^{\circ}$

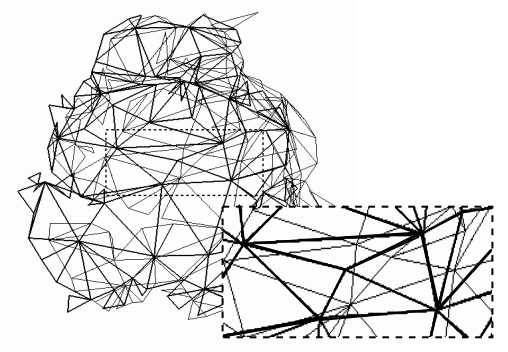

(c) Mismatch Figure 5. Smooth geometry and high approx-
imation error lead to sample mismatch.

\section{Shape Descriptor}

For each point $p_{i}$ in the target image, we want to find the best matching point $q_{j}$ in the reference image. This is a correspondence problem. Matching is easier if one uses a highly discriminating shape descriptor which diminishes the ambiguity in matching. Belongie and Malik proposed a descriptor for each image sample, called shape context [3]. This descriptor is composed of a set of vectors originating from a point $p_{i}$ to all other $\left(N_{s}-1\right)$ sample points, representing the overall shape relative to $p_{i}$. In principle, if the target and the reference images are coincident, finding a correspondence to $p_{i}$ in the reference image $D$ is equivalent to finding a point $q_{j}$ in $D$-image that possesses the same shape descriptor. In practice, two problems should be circumvented: the images are partially overlapped and the samples in the target and the reference images may be mismatched.

The solution for the first problem is to make use of the knowledge that the effects on a tetrahedron uniquely characterize an affine transformation and replace a shape 
descriptor with $\left(N_{S}-1\right)$ vectors by $C_{\left(N_{s}-1\right), 3}$ tetrahedrons whose sides are almost orthogonal. The three samples to which the vectors of a tetrahedron point at should lie inside the radial region limited by the minimal radius $R_{\min }=\max \left(D_{x}, D_{y}, D_{z}\right) / 5$ and the maximal radius $R_{\max }=R_{\min }+0.05 * \max \left(D_{x}, D_{y}, D_{z}\right)$, where $D_{x}, D_{y}$ and $D_{z}$ are the width, height, and depth of the bounding box of the image, as illustrates Figure 6. These radii have been obtained experimentally. The main idea underpinning them is to meet the trade-off between "covering as large as possible the overall shape" and "staying inside the overlapping region". In Section 6 we will show that with overlapping percentage more than $40 \%$, our implementation could successfully register the images that we tested.

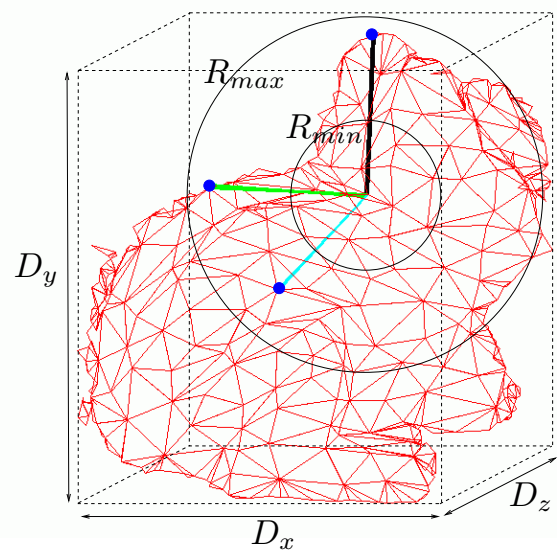

Figure 6. Shape descriptor.

The four image samples, at which the tetrahedron is anchored, may be further characterized by the differential geometric properties with respect to the mesh they belong. The geometric properties, such as principal curvatures and directions, may be estimated with the robust algorithm implemented by Rusinkiewicz [13]. These local attributes enrich the expression power of our proposed shape descriptor. It carries, in fact, some local and global information of the image shape. In order to enhance the estimation quality, we do not use the boundary samples for defining the origin of a shape descriptor.

Concerning the second problem, we will present a solution in Section 5.

\section{Matching}

Applying our proposed shape descriptor, the matching problem may be reduced to find the best correspondence among at most $N_{s} \times C_{\left(N_{s}-1\right), 3}$ possible shape descriptors in the decimated target mesh and at most $N_{D} \times C_{\left(N_{D}-1\right), 3}$ possible descriptors in the decimated reference mesh. To tackle this combinatorial explosion, we adopt, in analogous way, the conjunctive constraining conditions proposed by Chen et al. [7] in our matching procedure.

Let $S$-tetrahedron with samples $s_{i}, i=1,2,3,4$ be a shape descriptor of $S$-mesh and let $d_{i j}$ be the distance between the sample $s_{i}$ and sample $s_{j}$. A corresponding sample point $m_{1}$ of $s_{1}$ must be in the vicinity of the $s_{1}^{\prime}$ s correspondence in $D$-mesh and possesses similar differential geometric properties. Correspondences $m_{2}$ and $m_{3}$ to $s_{2}$ and $s_{3}$, respectively, are obtained in the same way as in the RBD algorithm with an additional constraint: the corresponding samples must be locally similar. And a sample $m_{4}$, that corresponds to $s_{4}$, must be the intersection of three spheres: the first centered in $m_{1}$ and with radius $d_{14}$, the second with center in $m_{2}$ and radius $d_{24}$ and the later, centered in $m_{3}$ and with radius $d_{34}$. Figure 7 illustrates the search spaces for the four correspondences in the decimated reference mesh.

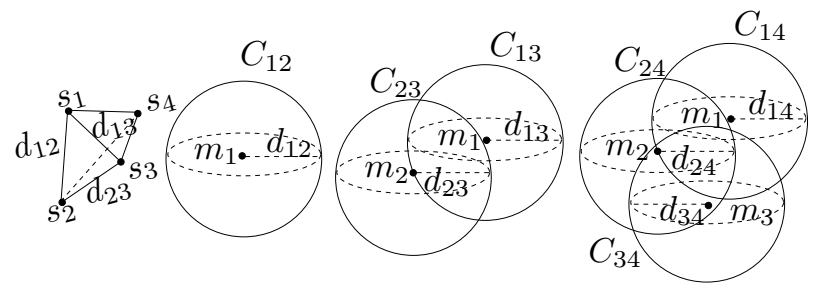

Figure 7. Pairwise matching.

As described, our proposed matching procedure may skip most of good correspondence candidates. This is because that the sample density is very low in the decimated meshes and that the samples in the target and reference meshes rarely are matchable. We suggest the following workaround that works satisfactorily: to enlarge the searching radius $d_{i j}$ by incrementing $2 s$. Figure 8 illustrates the matched shape descriptors, $\left(V_{s 1}, V_{s 2}, V_{s 3}\right)$ and $\left(V_{d 1}, V_{d 2}, V_{d 3}\right)$, from two distinct decimated meshes. From this pair we may compute one probable alignment transformation $\mathcal{T}_{S, D}$ from the expression

$\mathcal{T}_{S, D}=\left[\begin{array}{ccc}V_{d 1, x} & V_{d 2, x} & V_{d 3, X} \\ V_{d 1, Y} & V_{d 2, Y} & V_{d 3, Y} \\ V_{d 1, Z} & V_{d 2, Z} & V_{d 3, Z}\end{array}\right]\left[\begin{array}{ccc}V_{s 1, x} & V_{s 2, x} & V_{s 3, X} \\ V_{s 1, Y} & V_{s 2, Y} & V_{s 3, Y} \\ V_{s 1, Z} & V_{s 2, Z} & V_{s 3, Z}\end{array}\right]^{-1}$

Observe that although the angles between the vectors differ slightly, the estimated transformation matrix may make the target image correctly overlay the reference one.

It is worth remarking that encoding the local and global geometric restrictions in a shape descriptor allows us to handle the problem as a geometric one and applies the simple geometric algorithms for performing comparisons.

\section{Experimental Results}

In this section we present some results that corroborate our conjecture: faithful approximations to dense range im- 


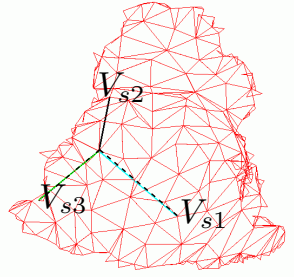

(a) $S$-mesh

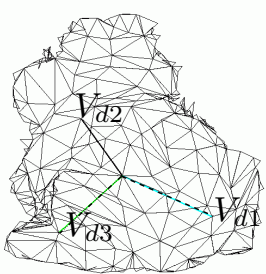

(b) $D$-mesh

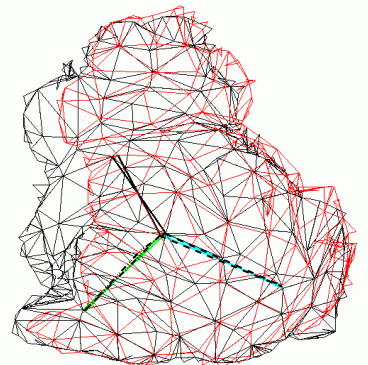

(c) $S$ over $D$
Figure 8. Matched shape descriptors.

ages may be used for finding a good correspondence estimate for two partially overlapping range data sets, and a shape descriptor aggregating local and global shape information is much more discriminant. In order to test our algorithm in several situations, we performed our experiments on range data from different repositories available on the internet $[12,16,15]$.

For evaluating the achieved registration quality, we selected three test sets with 9 range images in each: PITTPLANE, which contain plane regions (zero curvature) [16]; FROG with smooth geometry [16]; and ANGEL with several small protrusions [16]. Figure 9.a illustrates a pair $\mathcal{P}_{120-160}$ from the set FROG (view-angles, $120^{\circ}$ and $160^{\circ}$ ). We converted the point clouds to dense triangular meshes, according to the procedure suggested by Turk and Levoy [17], and simplified the meshes with, respectively, the approximation error $\epsilon=0.15 s, \epsilon=0.5 s$ and $\epsilon=s$, as explained in Section 3. Figure 9.b exemplifies the simplification result for $\mathcal{P}_{120-160}$ with $\epsilon=0.5 \mathrm{~s}$. Shape descriptors are built for each sample in the target decimated mesh. Next, to each shape descriptor the matching procedure given in Section 5 is carried out to find potential matchable candidates. Figure 9.c shows shape descriptors of a pair of matchable samples. All probable transformation matrices are, then, determined with the equation in Section 5 and evaluated in terms of their matching power. The best solution $\mathcal{T}$ is the one that overlay the largest region of the target mesh over the reference mesh (Figure 9.d).

The transformation matrix $\mathcal{T}_{i j}$ is pairwise determined for a pair of images $i$ and $j$. Using all the transformation matri-
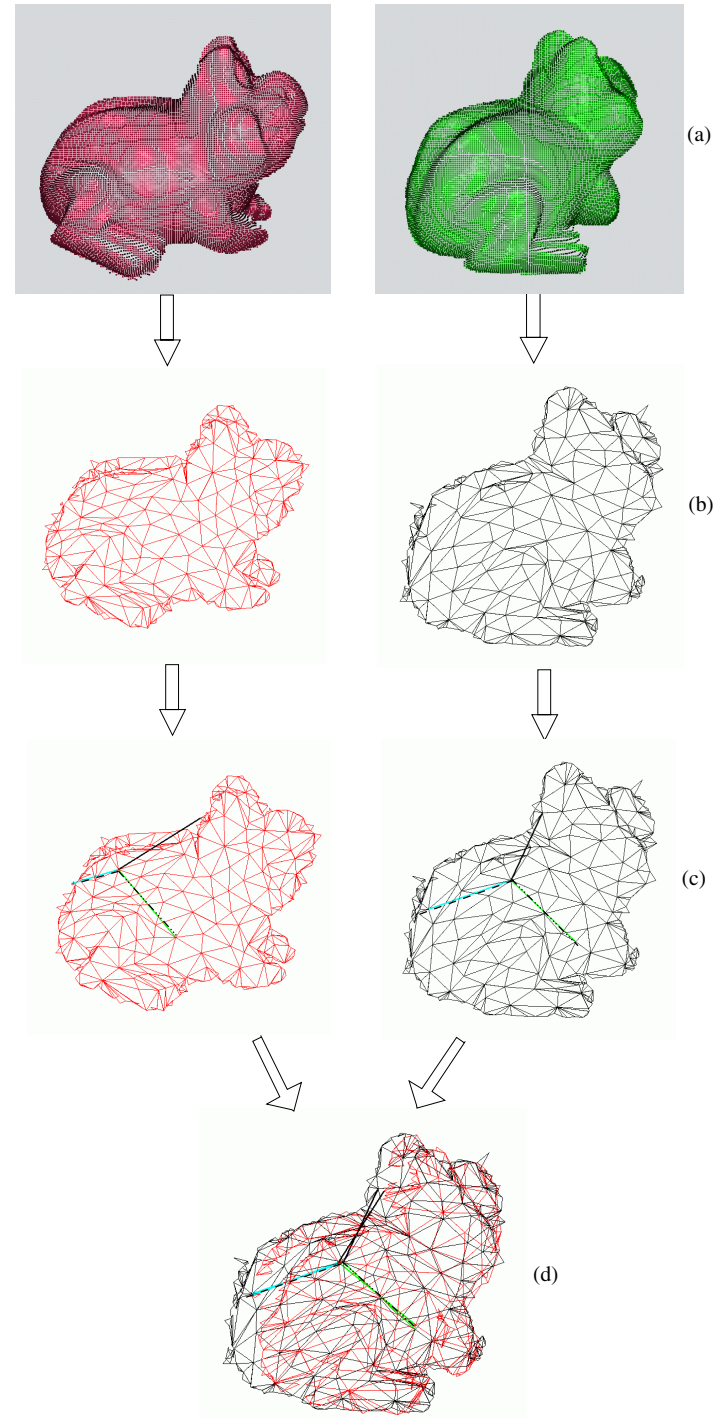

Figure 9. Pairwise matching flowchart.

ces as an initial guess, the iterative closest point algorithm (ICP) $[4,8]$, implemented in Scanalyze [14], iteratively refines them until the mean squared errors between the presumed correspondences are minimized. As a result, we get a globally consistent dense polygon mesh. Figure 10 presents the reconstructed models, accompanied by the average number of iterations ICP needed to converge. To visually evaluate the quality of the registration, each range image is plotted in a different color. The low number of iterations attests the matching power of coarse meshes.

In order to show the search space reduction capability of our proposal, we present in Table 1 the number of samples in distinct stages. In the column Samples we present the average number of samples $\mathcal{N}$ in the input range images. Without space reduction, we should exhaustively evaluate 


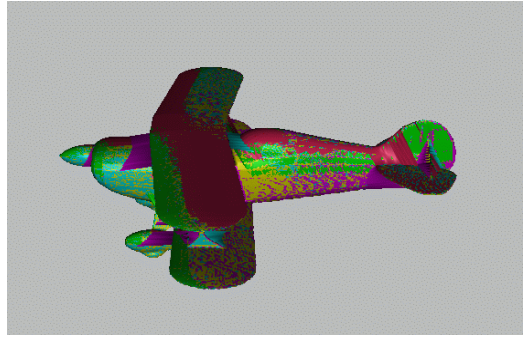

(a) 6 iterations

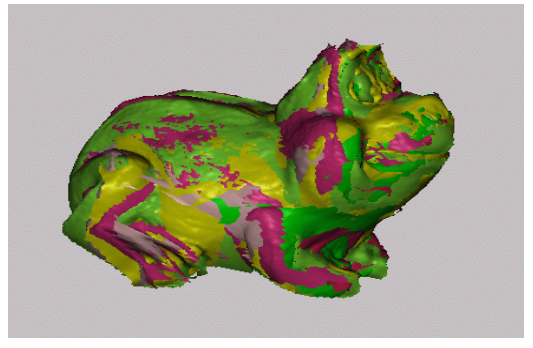

(b) 7 iterations

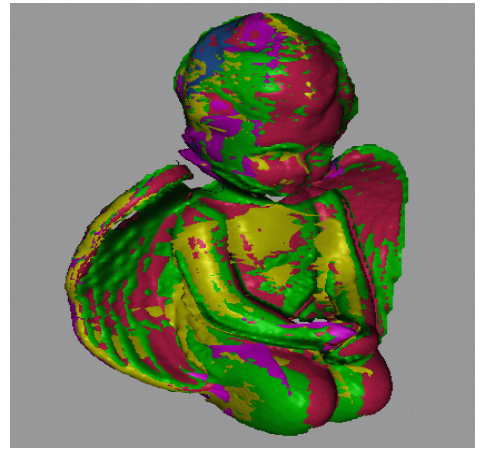

(c) 6 iterations

Figure 10. Assembled range images colored in distinct color: (a) PITT-PLANE; (b) FROG; and (c) ANGEL.

\begin{tabular}{|c|l|l|c|}
\hline Images & $\begin{array}{l}\text { Samples } \\
(\mathcal{N})\end{array}$ & $\begin{array}{l}\text { Vertices } \\
(N)\end{array}$ & Candidates \\
\hline PITT-PLANE set & 30712 & 1116 & 263467 \\
FROG set & 10223 & 449 & 25611 \\
ANGEL set & 12282 & 386 & 148293 \\
\hline
\end{tabular}

Table 1. Search space reduction

$\left(\mathcal{N} \times C_{(\mathcal{N}-1), 3}\right) \times\left(\mathcal{N} \times C_{(\mathcal{N}-1), 3}\right)$ possibilities to ensure that the best candidate is selected. After applying the QSLIM simplification algorithm, the average number of vertices $N$ in the decimated mesh is shown in the column Vertices. Nevertheless, instead of finding the best correspondence among average $\left(N \times C_{(N-1), 3}\right) \times\left(N \times C_{(N-1), 3}\right)$ possibilities for each pair of images, our matching algorithm further reduces the number of possibilities. The average potential matching transformations, that should in fact evaluated, is given in the column Candidates. Observe that the overall reduction ratio is more than $99 \%$. Despite this drastic sample space reduction, our algorithm is still able to deliver a coarse alignment transformation that makes the ICP algorithm converges in few iterations.

The second test is devised for evaluating the robustness of our algorithm to low overlapping region. For this test, we used more view pairs: 11 view pairs for the FROG and ANGEL sets and 5 view pairs for the PITT-PLANE set. The bar charts in Figure 11 show the average number of successful registrations with respect to the overlapping percentage of each matched view pair.

In the third test we evaluated the robustness of our proposed simplification paradigm to high decimation level. Our purpose is to validate our conjecture that it works well while the overall shape is preserved and the samples in the overlapping region are matchable. Fixing the overlapping percentage in $40 \%$, we additionally performed simplifica-

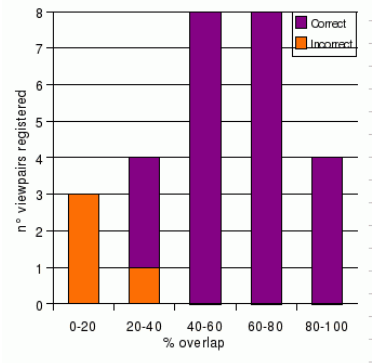

Figure 11. Successful registrations $\times$ overlapping percentage.

tions with two more approximation errors to all three test sets and evaluated their matching power in conjunction with the results obtained with the proposed value: one is less than the proposed one and another is greater. The bar charts in Figure 12 show the number of pairs have been successfully registered. Because the PITT-PLANE and FROG sets present smooth geometry, higher approximation error easily leads to loss of matchable samples in some view pairs, as explained in Section 3. If we reduce the approximation error, our algorithm presents better performance. For images with several small protrusions, as the ANGEL set, our proposed procedure presents better performance.

\section{Concluding Remarks}

In this paper we present a technique for an automatic registration of two partially overlapping range images on the basis of their simplified triangular meshes. The most important contribution of our work for crude registrations is to use a faithful approximation of the dense range data to reduce the search space. Among the advantages of this approach, 

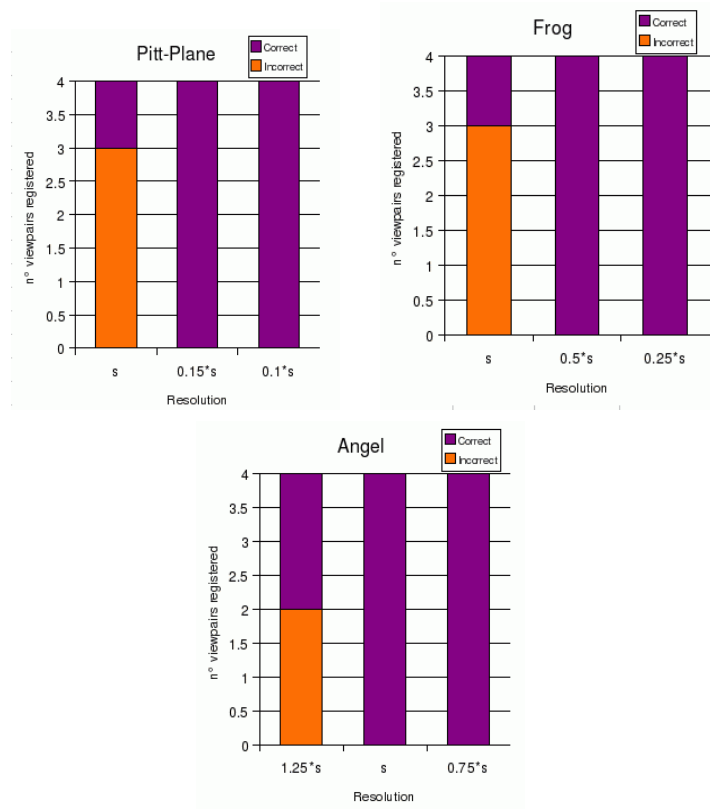

Figure 12. Successful registrations $\times$ level of decimation.

we may list: (1) drastic reduction of the input data, without missing the relevant features and (2) easiness in constructing discriminant structures that integrate two intrinsic properties: distance and curvature. Experimental results show that our algorithm is accurate and efficient. Numerical data attest that our proposal can reduce, in average, the search space more than $99 \%$. This allows us to exhaustively evaluate all the transformation candidates and to choose the best one.

Decimation is the most important step in our proposed crude registration algorithm. We have adapted the available QSLIM simplification algorithm. The workaround may, however, degrade the performance. For ensuring that samples are spatially close in the overlapping regions we cannot use meshes with very low level of detail. We would like to investigate a simplification algorithm that takes into consideration the relative placement of a contraction vertex.

\section{Acknowledgments}

We would like to acknowledge FAPESP for financial support under the grant numbers 00/10913-3 and 2003/13090-6.

\section{References}

[1] A.Johnson and M.Hebert. Using spin images for efficient object recognition in cluttered $3 \mathrm{~d}$ scenes. IEEE Transactions on Pattern Analysis and Machine Intelligence, 21:433-448, 1999.

[2] A. Ashbrook, R. Fisher, N. Werghi, and C. Robertson. Aligning arbitrary surfaces using pairwise geometric histograms. In Proceedings of Noblesse Workshop on Nonlinear Model Based Image Analysis, pages 103-108, 1998.

[3] S. Belongie and J. Malik. Shape matching and object recognition using shape contexts. In IEEE Transactions on Pattern Analysis and Machine Intelligence, volume 24, pages 509-522, 2002.

[4] P. Besl and N. McKay. A method for registration of 3-D shapes. IEEE Transaction on Pattern Analysis and Machine Intelligence, 14(2):239-256, 1992.

[5] B.M.Planitz, A. Maeder, and J. Williams. The correspondence framework for $3 \mathrm{~d}$ surface matching algorithms. Computer Vision and Image Understanding, 97:347-383, 2005.

[6] B.M.Planitz, A. Maeder, and J. Williams. Using the correspondence framework to select surface matching algorithms. In Proceedings of the Australian Pattern Recognition Society (APRS) Workshop on Digital Image Computing, pages 85-90, 2005.

[7] C.-S. Chen, Y.-P. Hung, and J.-B. Cheng. RANSAC-based DARCES: A new approach to fast automatic registration of partially overlapping range images. IEEE Transactions on Pattern Analysis and Machine Intelligence, pages 12291234, 1999.

[8] Y. Chen and G. Medioni. Object modeling by registration of multiple range images. Image and Vision Computing, pages 145-155, 1992.

[9] M. Garland and P.Heckbert. Simplification using quadric error metrics. In Computer Graphics, volume 31, pages 209216, New York, USA, 1997.

[10] P. Krsek, T. Pajdla, and V. Hlavác. Differential invariants as the base of triangulated surface registration. Computer Vision Image Understanding, 87:27-38, 2002.

[11] M.A.Fischler and R.C.Bolles. Random sample consensus: A paradigm for model fitting with aplications to image analysis and automated cartography. Comm. Association for Computing Machinery, 24(6):381-395, 1981.

[12] Range images. http://sampl.eng.ohio-state. edu/sampl/data/3DDB/RID.

[13] S. Rusinkiewicz. Estimating curvatures and their derivatives on triangle meshes. In 2DPVT'04: Proceedings of the $3 D$ Data Processing, Visualization, and Transmission, 2nd International Symposium on (3DPVT'04), pages 486-493, Washington,DC,USA, 2004.

[14] Scanalyze, a system for aligning and merging range data. http://graphics.stanford.edu/software/ scanalyze.

[15] Range images. http://graphics.standford. edu/data/3Dscanrep.

[16] Range images. http://range.informatik. uni-sttutgart.de/htdocs/html.

[17] G. Turk and M. Levoy. Zippered polygon meshes from range images. In Computer Graphics Proceedings, pp. 311-318. Florida, 1994. Siggraph 94.

[18] J. Vanden and L. Gool. Automatic crude patch registration. Computer Vision Image Understanding, 87, 2002. 